\title{
Measuring adulterants in street drugs: Implementation of drug residue testing using portable mass spectrometry in a Northeastern U.S. harm reduction program, 2019-2020
}

\author{
Mathieu Boulad \\ Tufts University School of Medicine \\ Atsushi Matsumoto \\ Northeastern University \\ Claudia Santelices \\ Northeastern University \\ Thomas J. Stopka ( $\square$ thomas.stopka@tufts.edu ) \\ Tufts University School of Medicine
}

\section{Research Article}

Keywords: Opioids, fentanyl, portable mass spectrometry, adulterants, Northeastern U.S.

Posted Date: December 10th, 2021

DOI: https://doi.org/10.21203/rs.3.rs-1152519/v1

License: (c) (1) This work is licensed under a Creative Commons Attribution 4.0 International License.

Read Full License 


\section{Abstract}

Background: Fatal opioid overdose deaths involving illicitly manufactured fentanyl continue to escalate in the U.S. Drug checking services, as a harm reduction intervention for people who use drugs, has gained support as an effective strategy to reduce fatal overdoses. We examined implementation of drug checking services using portable devices in a syringe services program in the Northeastern U.S.

Methods: Trained staff collected trace drug specimens from used paraphernalia provided by participants who requested drug checking services. All the specimens were tested using a portable mass spectrometer and sub-samples were tested for the detection of fentanyl using fentanyl testing strips. We assessed characteristics of participants who used drug checking services, self-reported types of trace specimens of substances that participants reported providing for testing, the actual mass spectrometer test results of these specimens, and agreement of the mass spectrometer and fentanyl testing strips results in detection of fentanyl and fentanyl analogues.

Results: Of 155 unique participants who provided demographic information, 59\% identified as male and $74.1 \%$ as White, with a mean age of 37.7 years. Based on analysis of 396 specimens tested with the portable mass spectrometer, the most common single substance detected was fentanyl (37.7\%), without a trace of heroin or other adulterants, followed by methamphetamine (18.2\%), and cocaine (13.6\%). Fentanyl and fentanyl analogues were detected in specimens provided by participants that were reported as heroin $(60.8 \%)$, cocaine $(11.1 \%)$, and methamphetamine $(6.7 \%)$. We found modest agreement of testing results between the mass spectrometer and fentanyl testing strips.

Conclusions: Use of drug checking services within syringe services programs is in its initial test stages. Knowledge about the contents of substances purchased, and conversations between syringe services program participants and staff, have the potential to facilitate informed decisions to decrease overdose risks through engagement in harm reduction strategies. Through analysis of newly implemented drug checking services, we noted participant characteristics and dissonance between participants' reports of the trace drug specimens submitted for testing and the actual drugs and adulterants detected by mass spectrometer results, which has implications for overdose risk, highlighting opportunities for harm reduction responses.

\section{Background}

Opioid overdoses are at crisis levels in communities across the US, with more than 93,000 fatal opioid overdoses in 2020 (1-7), worsening during the COVID-19 pandemic, with reports of an estimated 100,306 overdose deaths from April 2020 to April 2021 (8-10). Synthetic opioids such as fentanyl and fentanyl analogues are reported as the most prominent contributors of these overdose deaths $(3,11)$. Fentanylrelated overdose rates increased from 0.3 per 100,000 in 1999 to 9.9 in 2018, a 33-fold increase (1). Similar trends have been observed in Massachusetts, where opioid overdose mortality rates quintupled from 5.9 per 100,000 in 2000 to 30.2 per 100,000 in 2020, with more than 2,000 opioid-related overdose 
deaths per year between 2016 to 2020 (11), and an increase in the presence of fentanyl in toxicology reports for opioid-related overdoses increased from $41.8 \%$ of opioid overdoses in 2014 to $92 \%$ in 2020 (12).

With the diffusion of fentanyl in the illicit drug supply across the U.S. in recent years, and the high rates of overdoses tied to fentanyl-adulterated substances $(13,14)$, drug checking has gained support as an effective strategy to reduce fatal overdoses (15-17). As such, many harm reduction programs have incorporated distribution and use of fentanyl test strips (FTS) into their wrap-around services for people who use drugs (PWUD), and have demonstrated changes in behaviors among their participants as a result of positive fentanyl tests. These changes include: plans to decrease the dose used, discarding a drug entirely, using with someone else nearby, or keeping naloxone on hand, which are consistent with principles of harm reduction (18-20). Research has also shown that FTS provide valuable information to people who use opioids (18-20), and while sensitive and easy to use in the field, the information obtained from them is fairly limited insofar as they only report on the presence or absence of fentanyl and some analogues (21). Given that the presence of fentanyl is ubiquitous in most street-purchased opioids, and is increasing in other drugs obtained in the community $(22,23)$, more sophisticated drug checking approaches are needed to inform harm reduction staff and their clients about amplified and unpredictable overdose-related risks.

In recent years, drug- and adulterant-detecting technologies have been developed in such a way that devices are now able to quantify the components of drug specimens, and they are easier to use in the community $(21,24,25)$. Devices such as portable mass spectrometers have the potential to amplify drug testing capabilities and related harm reduction messages and approaches $(21,26)$. Being able to test specimens in real time, while attending syringe services programs (SSPs) or other community-based agencies, can offer data-informed harm reduction messages to reduce risks based on testing results. Use of two drug checking devices including the portable MX908 Mass Spectrometer and FTS was initiated at a Northeastern U.S. SSP during early-2019 and early-2020, respectively. The overarching goal of our study was to evaluate the implementation, use, and results from the portable MX908 Mass Spectrometer (MX908) (27) and FTS (28) in a harm reduction setting. We analyzed SSP data to assess the demographic characteristics of the clients who chose to have their drug paraphernalia tested for trace substances and adulterants, compare the substances that clients believed they were testing to the tests results from drug checking services (DCS), and compare preliminary results from the MX908 device and FTS.

\section{Methods}

\section{Testing devices}

The MX908 device was used to test all the trace specimens collected by SSP staff. The MX908 is a portable handheld device that uses high pressure mass spectrometry to analyze chemical properties of a sample. Testing offers information on presence, amount, and combinations of drugs or adulterants 
detected in a sample (27) and, to our knowledge, this is the first study to assess the use of the MX908 device in DCS within a fixed-site SSP in the U.S. Sub-samples of the specimens were also tested with FTS per SSP staff's discretion. FTS is a rapid immunoassay test designed to detect the absence or presence of fentanyl and fentanyl analogues. FTS does not provide information on concentration or types of fentanyl analogues detected. Although off-label use of FTS has not been approved in the U.S., FTS has been implemented as a harm reduction intervention in $\operatorname{SSPs}(29,30)$.

\section{Specimen collection}

Following a manufacturer-provided training of SSP staff to use the MX908 device in a community based SSP, staff began to receive drug packaging and used paraphernalia (e.g., baggies, vials, cookers, cottons) from SSP clients that contained trace elements of substances. SSP staff collected basic data elements from clients upon drop-off of trace specimens. Measures included: client demographics and specimen details (type, suspected substance purchased, effect of substance when used, date of collection, location of collection). Data were collected on a paper insert attached to the specimen and were then manually entered into a Microsoft Excel spreadsheet.

\section{Study measures}

Data collected were self-reported by participants and recorded by trained staff involved in DCS with the MX908 and FTS, during the provision of SSP services.

Demographic data for participants who submitted trace specimens for DCS included self-reported measures for: date of birth, which we used to calculate the age of the participants based on the day they submitted trace specimens for testing; gender (male, female, or transgender); and race/ethnicity (Black, White, Latinx, Other).

Trace specimens were detected by taking a swab of drug paraphernalia (e.g., from the inside of an empty baggy). The swab was then rubbed on three MX908 test strips, which were ultimately inserted, one-afterthe other into the MX908 device for scanning. If the first scan produced definitive results, subsequent scans were not needed. If the first scans were indeterminant, or did not produce results, the $2^{\text {nd }}$ and $3^{\text {rd }}$ scans were conducted. Specimen measures included the participants' self-report of the trace specimens they were testing (e.g., "Dope", "China", "Brown", and "Fire" were interpreted as Heroin; "Tina" was interpreted as Methamphetamine; Xanax, alprazolam, diazepam, and "benzos" were collapsed and defined as benzodiazepine).

DCS metrics consisted of objective MX908 and FTS testing results recorded by trained SSP staff and included the read-out from the spectrometer, the number of tests attempted, differing results, and additional notes from participants and staff during DCS such as: "Huge rush, had legs, wasn't sick", "Client obtained liquid while doing a date, was worried it was a date rape drug", "Felt poisonous, went to ER [Emergency Room]", and "2 ODs on [location Ave.] that day on the same supply." The read-out options 
from the MX908 spectrometer were based on the library of adulterants programmed into the device and updated periodically by the manufacturer.

For the current analysis of MX908 results, we established decision rules to facilitate consistency across testing result read-outs from the MX908: 1) Test results yielding "Red" alarms, which indicated detection of a drug or an adulterant, were included in the analysis; 2) tests results presenting "Yellow" alarms were not included for analysis as they were tied to safety precautions for testers and did not indicate definitive detection of a drug or an adulterant; 3 ) test results that did not have a designated color-coded alarm were assumed to be "Red" and were included in the final analysis; 4) when a trace specimen was tested more than once, all "Red" alarms across testing instances were concatenated and included in the analysis, regardless of number of attempts or differing reads with repeat testing; 5) when test results yielded a coalarm for "Hydromorphone," which was usually detected with a "Yellow" alarm, and "Cocaine", which was usually detected with a "Red" alarm, only the latter was retained and included in analysis; 6) test results yielding a substance with a "Yellow" alarm were designated as "Not/Applicable"; 7) test results yielding "No Target" or "No Signal" reads were interpreted as "Not/Applicable"; 8) test results labeled "Acetylfentanyl", "Carfentanyl", "Furanylfentanyl", "Butyrylfentanyl" and "Methylfentanyl" were aggregated into a "Fentanyl Analogues" category: and 9) test results for "U-47700" and "AH-7921" were aggregated into a "Synthetic Opioids" category. Additional metrics included the testing results of these same trace specimens, using FTS, which were recorded as either "Positive" or "Negative".

\section{Data analysis}

We analyzed data associated with an initial set of 396 trace specimens tested with the MX908, 191 of which were also tested with FTS, from February 2019 to March 2020. To analyze the data, we standardized and formatted entries for reported specimen testing results.

For demographic characteristics of the 155 confirmed non-duplicated participants, we calculated measures of central tendency (means, standard deviation, median, interquartile range), as well as percentages and rates, for all variables. Descriptive statistics were calculated in Microsoft Excel 2019.

The study protocol was reviewed and approved by the [blinded] Institutional Review Board.

\section{Results}

\section{Demographic information}

The mean age of non-duplicated participants who had their specimens tested was 37.7 years ( $s d=9.02$; range $=18-59)$. Participants were predominantly male (59.0\%) and White (74.1\%) (Table 1$)$.

Table 1. Demographic characteristics of clients who had their specimens tested with the MX908 mass spectrometer $(n=155) .{ }^{1}$ 
Characteristics $\quad n \quad \%$

\begin{tabular}{|c|c|c|}
\hline $\begin{array}{l}\text { Age (years) } \\
\text { Mean } \\
\text { (Std. Dev.) }\end{array}$ & $\begin{array}{l}110 \\
37.7(9.02)\end{array}$ & - \\
\hline Gender $(n=144)$ & & \\
\hline Male & 85 & $(59.0 \%)$ \\
\hline Female & 58 & $(40.3 \%)$ \\
\hline Transgender Male to Female & 1 & $(0.7 \%)$ \\
\hline Race/Ethnicity $(n=139)$ & & \\
\hline White & 103 & $(74.1 \%)$ \\
\hline Latinx & 23 & $(16.5 \%)$ \\
\hline Black & 10 & $(7.2 \%)$ \\
\hline Other & 3 & $(2.2 \%)$ \\
\hline
\end{tabular}

${ }^{1}$ Change in sub-sample size for demographic characteristics due to missing responses

\section{Drug type by trace specimens provided for DCS}

Among the 396 DCS instances, participants reported the type of trace drug specimen they provided for testing in 342 instances (86.4\%). Of these, more than half of the tested specimens were from participants who provided trace specimens that they reported were heroin (52.9\%), while less than one in five participants (17.5\%) provided trace specimens that they reported were methamphetamine. Finally, $16.6 \%$ of specimens submitted for testing were from participants who provided trace specimens that were believed to contain fentanyl (Table 2a).

\section{MX908 specimen testing results}

The MX908 device was used to test 396 specimens. Substances were detected by the MX908 in 324 instances (81.8\% match rate to a substance in the MX908 library), and 72 events were unsuccessful, or did not match a substance in the library. The following results focus on the final analytical sample of specimens $(n=324)$. In non-aggregated MX908-detected specimen categories, fentanyl, with no trace of heroin or other substances in the MX908 library, was detected exclusively in nearly 4 of $10(37.7 \%)$ specimens. Additionally, the second most common MX908 single result was "Methamphetamine", with $18.2 \%$ testing positive, followed by cocaine (13.6\%) (Table $2 b$ ).

Table 2 Self-reported substance type by trace specimens provided for DCS and nonaggregated MX908 specimen results 


\section{Table 2a.}

Self-report of substance type by trace specimens provided for

DCS* $(n=342)$

Heroin

$181(52.9 \%)$

Methamphetamine

60

Fentanyl

$35 \quad(10.2 \%)$

Heroin/Fentanyl

20

Crack/Cocaine

18

Benzodiazepine

13

Fentanyl/Cocaine

2

Heroin/Cocaine

Bupropion

Other $^{1}$

Table 2b.

MX908 specimen results $(n=324)$

Fentanyl

Methamphetamine

Cocaine

Fentanyl/Fentanyl Analogue

Cocaine/Hydrocodone

Heroin

12

(3.7\%)

Fentanyl Analogue

14

Methamphetamine/Fentanyl

Cocaine/Fentanyl

Fentanyl and Analogue Combinations ${ }^{2}$

8

25

Other $^{3}$

$\mathrm{N} / \mathrm{A}(\mathrm{n}=396)$

* Drug checking services

1 Includes drugs reported as being combined substances

2 Includes testing results detecting combinations of fentanyl or fentanyl analogues not listed above such as:

"Heroin/Fentanyl", "Fentanyl Analogue/Synthetic Opioids", "Methamphetamine/Fentanyl/Fentanyl

Analogue/Acetaminophen"

3 Includes testing results detecting non-fentanyl/analogue containing combinations not listed above, such as:

"Acetaminophen", "MDMA/Caffeine", "Methamphetamine/Cocaine/Scopolamine", "Synthetic Opioids"

In summarizing aggregated trace specimen categories detected, more than half of all MX908 results included an opioid (61.4\%), either alone or in combination with other substances, and among these, and $55.6 \%$ of test results noted the presence of fentanyl, alone or in combination with another substance (Table 3). Close to one in four tests $(22.1 \%)$ detected methamphetamine, alone or in combination with another drug, and $17.5 \%$ of specimens tested positive for cocaine. 
Table 3. Aggregated categories of substance-specific results from MX908-tested trace specimens $(n=324)$.

\begin{tabular}{ccc} 
Substance Detected & $\boldsymbol{n}$ & $\%$ \\
\hline Opioids $^{1}$ & 199 & $61.4 \%$ \\
Fentanyl $^{2}$ & 180 & $55.6 \%$ \\
Heroin & 18 & $5.6 \%$ \\
Hydrocodone & 2 & $0.6 \%$ \\
Cocaine & 61 & $17.5 \%$ \\
\hline Methamphetamine & 77 & $22.1 \%$ \\
\hline
\end{tabular}

1 "Opioids" category includes fentanyl, fentanyl analogues, heroin, hydrocodone, and synthetic opioids

2 "Fentanyl" category includes fentanyl and fentanyl analogues

\section{Participant-reported versus actual trace substance detected in MX908 test results}

Out of 181 submitted specimens that were reported by participants as heroin, $60.8 \%(n=110)$ contained some form of fentanyl or fentanyl analogue, according to MX908 results, while $7.7 \%(n=14)$ contained heroin, including heroin alone $(n=12)$, or heroin in combination with non-fentanyl adulterants $(n=2)$ (Table 4). Conversely, if a participant provided a trace specimen reported to be methamphetamine $(n=60)$, or any fentanyl combination ( $n=57)$, the MX908 confirmed these in $71.7 \%(n=43)$ and $68.4 \%(n=39)$ of testing events, respectively. Among participants who submitted non-opioid specimens, such as methamphetamine or crack/cocaine trace specimens $(n=78), 7.6 \%(n=6)$ tested positive for fentanyl or a fentanyl analogue.

Table 4. Comparing MX908 test results with self-reported specimen type provided by participants for DCS* $(n=324)$ 


\begin{tabular}{|c|c|c|c|c|c|}
\hline & & \multicolumn{4}{|c|}{ Self-reported specimen type provided by participants for DCS } \\
\hline & & Heroin & Methamphetamine & Crack/Cocaine & $\begin{array}{c}\text { Fentanyl } \\
\text { in any combination }\end{array}$ \\
\hline \multirow{3}{*}{$\begin{array}{l}\text { Compared } \\
\text { to results } \\
\text { from } \\
\text { MX908 }\end{array}$} & $\begin{array}{l}\text { Count } \\
\text { (specimen provided) }\end{array}$ & 181 & 60 & 18 & 57 \\
\hline & $\begin{array}{l}\text { Actually contained: } \\
\text { (per MX908 Results) }\end{array}$ & $\begin{array}{c}14^{1} \\
(7.7 \%)\end{array}$ & $\begin{array}{c}43^{2} \\
(71.7 \%)\end{array}$ & $\begin{array}{c}13 \\
(72.2 \%)\end{array}$ & $\begin{array}{c}39^{5} \\
(68.4 \%)\end{array}$ \\
\hline & $\begin{array}{l}\text { Included fentanyl/fentanyl } \\
\text { analogue as an adulterant } \\
\text { (per MX908 Results) }\end{array}$ & $\begin{array}{c}110 \\
(60.8 \%)\end{array}$ & $\begin{array}{c}4^{3} \\
(6.7 \%)\end{array}$ & $\begin{array}{c}2^{4} \\
(11.1 \%)\end{array}$ & Same as above \\
\hline
\end{tabular}

${ }^{*}$ DCS: Drug checking services

${ }^{1}$ Included I each of "Heroin/Methamphetamine", "Heroin/Cocaine"

${ }^{2}$ Included 1 each of "Methamphetamine/MDMA", "Methamphetamine/Amphetamine"

"Included I"Cocaine/Benzodiazepine"

${ }^{4}$ An additional 7 trace specimens included Cocaine/ Hydrocodone combination, not included in the analysis

"An additional 5 trace specimens that had "N/A" results on the $M X 908$ tested positive for "Fentanyl" with the FTS, bringing the potential \% of fentanyl to $77.19 \%$

\section{Agreement of MX908 testing results with FTS}

Within the initial set of 396 specimens tested with the MX908 device, 191 (48.2\%) were also tested for fentanyl using FTS. Among the 180 specimens that tested positive for fentanyl and fentanyl analogues with MX908 analyses, $72(40.0 \%)$ were also tested using FTS. Sixty of the 72 specimens $(83.3 \%)$ tested with FTS tested positive for fentanyl/fentanyl analogues and 12 specimens (16.7\%) tested negative using FTS (Table 5).

Among 144 specimens that were tested with the MX908 device and came back negative for fentanyl/fentanyl analogues, 78 (54.2\%) were also tested using FTS. Slightly more than one-third (34.6\%; $\mathrm{n}=27)$ tested positive for fentanyl/fentanyl analogue and $51(65.4 \%)$ tested negative.

Among 72 specimens that were tested with the MX908 device and came back as having No Signal/No Target, 47 (65.3\%) were also tested using FTS. Approximately four in $10(40.4 \% ; n=19)$ tested positive for fentanyl/fentanyl analogue while 6 in $10(59.6 \%$; $=28)$ tested negative.

Table 5 Comparing fentanyl test results from the MX908 device $(n=396)$.

\begin{tabular}{|c|c|c|c|c|}
\hline MX908 Testing Entries $(n=396)$ & \multicolumn{3}{|c|}{ (FTS) Testing for Fentanyl/Analogues ( $n=191)$} & \multirow[b]{2}{*}{ Concordance } \\
\hline Results & Positive & Negative & Total & \\
\hline Fentanyl and/or Fentanyl Analog & \multirow[t]{2}{*}{60} & \multirow[t]{2}{*}{12} & \multirow[t]{2}{*}{72} & \multirow[t]{2}{*}{$83.3 \%$} \\
\hline 180 & & & & \\
\hline Other (Non fentanyl results) & \multirow[t]{2}{*}{27} & \multirow[t]{2}{*}{51} & \multirow[t]{2}{*}{78} & \multirow[t]{2}{*}{$65.4 \%$} \\
\hline 144 & & & & \\
\hline N/A (No Target or No Signal) & \multirow[t]{2}{*}{19} & \multirow[t]{2}{*}{28} & \multirow[t]{2}{*}{47} & \\
\hline 72 & & & & \\
\hline Total & \multirow[t]{2}{*}{106} & \multirow[t]{2}{*}{91} & \multirow[t]{2}{*}{197} & \multirow[t]{2}{*}{$74.0 \%$} \\
\hline 396 & & & & \\
\hline
\end{tabular}




\section{Discussion}

To our knowledge, this is the first study to report drug specimen testing with the portable MX908 Mass Spectrometer in the community through an SSP located in the Northeastern U.S. Our novel study provides the demographic characteristics of SSP clients who were willing to utilize DCS, types of trace specimens submitted by SSP clients for testing, and the testing results from MX908 and FTS.

Our descriptive analyses allowed us to obtain an initial understanding of the characteristics of the people who used the DCS at an SSP. We found that testing participants were largely White $(74.1 \%)$ and male $(59.0 \%)$, with a median age of 37 years, similar to participant characteristics in other Northeastern U.S. communities, as described in a study designed to assess acceptability of community-based DCS (31). We compared the racial and ethnic composition of SSP clients who were willing to use DCS to overall SSP clients who accessed any services at the same SSP during roughly the same timeframe that our study was conducted. We found overrepresentation of White SSP clients and underrepresentation of Black, Latinx and Other SSP clients among participants who utilized DCS, suggesting a need for outreach efforts designed to improve access to DCS among Black, indigenous, and people of color who receive SSP services. The need to better support these underrepresented communities is further highlighted in previous studies that have shown disproportionate criminalization and insufficient harm reduction efforts within black communities (32).

Among the individuals who opted to use DCS in our study, $68.9 \%$ provided trace specimens that they reported to be opioids for testing, and close to a quarter of participants provided trace specimens reported to be stimulants such as crack/cocaine or methamphetamines. Use of DCS by a substantial portion of participants who provided stimulant specimens for testing may reflect a combination of the ongoing effects of the opioid crisis, with the most recent fourth wave of the crisis, in which people who primarily use methamphetamine or cocaine, or a mix of non-opioid drugs are encountering increasing amounts of opioids or fentanyl adulterants in their drug supply (32-35). These findings align with recent behaviors reported among PWUD in another study which tested stimulants or presumed non-opioid substances for possible adulterants. Tupper et al., using a Fourier Transform Infrared Spectrometer for DCS in a Canadian site, reported close to $15 \%$ of specimens submitted for testing that were expected to be methamphetamine, noting potential concerns for adulteration of stimulants (29).

We found that over half of the tested specimens had positive results for fentanyl or fentanyl analogues. In the setting of well documented increases in fentanyl related overdoses $(1,3,11)$ and high rates of fentanyl detection by FTS strips $(17,19)$, this is hardly surprising. What does stand out in these reported findings, however, is that the single most common MX908 result was the detection of fentanyl alone, with no other adulterants in over a third of the testing results. Additionally, heroin in any combination was only detected in $5.6 \%$ of testing results. Taken together, these results help to describe the local drug landscape, which consists predominantly of fentanyl, and most often with no additional illicit adulterants, and a dwindling presence of heroin $(36,37)$. On a more participant-centric level, using DCS with FTS to inform participants about the presence of fentanyl in their submitted specimen has been shown to be an 
effective risk mitigating strategy as demonstrated in previous studies $(15,16,30,31,38)$. Further testing with devices such as the MX908, which can provide participants with more details on the trace elements present in the substances that they obtain and use, could be an even more powerful strategy in risk mitigation than FTS alone. Another implication of these findings is that they might reflect increasing levels of opioid dependence and tolerance, given that most drugs used in this environment contain fentanyl, and often exclusively fentanyl as the illicit substance detected. Consequently, increasing opioid tolerance in local substance-using populations aligns with recently documented increases in both overdoses and the number of naloxone kits needed to reverse an overdose (39). In this vein, our results indicated that over half $(52.9 \%)$ of the participants reported their specimens as heroin and, more importantly, $16.6 \%$ reported fentanyl alone or in combination with heroin or cocaine. Perhaps participants testing specimens that they believed to be fentanyl reflects an increase in opioid tolerance and dependence that is difficult to satisfy with heroin alone, leading to fentanyl seeking behaviors in drug use $(36,37,39,40)$. Another possibility is that those reporting specimens as heroin, might have been using the term as a catch all reference to opioids, including fentanyl, as has been previously reported (41).

An important element of these results was the discrepancy between participants' self-report of the specimens they obtained and actual MX908 test results. Previous studies reported on the ability of PWUD to discern whether the drugs they obtained locally contained fentanyl or heroin based on color, taste, and other gross characteristics or subjective experiences $(22,41,42)$. Our findings point to the challenges in accurately detecting actual substances through these senses alone. Most striking in our findings was the discrepancy between reported heroin as a presumed substance with the majority of results detecting fentanyl or fentanyl analogues, and less than $6 \%$ actually detecting heroin. Discrepancies between perceived vs. actual substances used place PWUD at significant risk for overdose from fentanyl. While discrepancies were less notable among participants who tested what they reported as crack/cocaine and methamphetamine, the risks of overdose in opioid naïve PWUD is especially concerning, as reported in early descriptions of the fourth wave of the opioid overdose epidemic (32-35). Perhaps drug checking services have a particularly important role among PWUD who do not use opioids. While FTS might be sufficient to detect fentanyl, additional testing such as that possible with the MX908, and other portable electronic devices, could help in detecting other synthetic opioids and fentanyl analogues that may not yield a positive FTS.

Comparing the MX908 testing results to FTS is a critical element in this study given that FTS has been identified as a reliable and valid device to detect the presence of fentanyl (16). We found that, in more than $83 \%$ of the cases, MX908 detection of fentanyl/fentanyl analogues in a specimen agreed with FTS and $65 \%$ of the cases of MX908 non-detection of fentanyl/fentanyl analogues agreed with FTS. There does appear to be some discordance in the non-detection of fentanyl/fentanyl analogues in the MX908 results compared to the FTS.

Our findings from preliminary results should be interpreted while considering several limitations. First, MX908 testing was conducted during the initial stages of use of the device, and there was a steep 
learning curve in the proper use of the device, as well as the interpretation of testing results including classifying alarms and co-alarms. SSP staff used FTS testing strips when available, to cross check MX908 testing results for the presence of fentanyl and evaluate possible inconsistencies. Several technical challenges and malfunctions of the MX908 device were identified by SSP staff, which hindered initial implementation and the consistent use. These challenges included: the clogging of the equipment; troubleshooting episodes as a result of inconsistency in the amount of trace specimens required for testing; and false positive test results. Second, all the specimens provided by participants for testing were at risk of cross contamination given that drug paraphernalia could be reused. Third, the current study implemented DCS in a community based SSP located the Northeastern, U.S. Thus, our findings may not be generalizable to harm reduction agencies in other regions. Fourth, our analysis of demographic statistics relied on data collected from a subgroup of non-repeat participants, as confirmed by the SSP, who responded to demographic questions. There was missing demographic information from some participants who received DCS and others could not be confirmed as unique or non-repeat participants and as such were not included in the analysis of demographic. Therefore, the total number of unique participants in the current study was likely higher than the number we reported, and it is possible that some subpopulations of color had better representation. Fifth, while participants may report a submitted specimen as being "heroin", "dope" or "brown", they may be using these terms as a catch-all street term for opioids in general, not necessarily believing that these specimens are devoid of any fentanyl. Lastly, the library of substances to be detected by MX908 was updated periodically throughout the study period; consequently, detection of some substances and adulterants in specimens might have been missed in the early phase of the implementation of DCS.

\section{Conclusion}

Implementation of DCS in the opioid crisis can better inform understandings of changes and fluctuations in a local drug landscape, while at the same time, providing critical information about contents of drugs to support tailored harm reduction and drug overdose prevention conversations between SSP staff and PWUD. We assessed the demographic characteristics of the SSP participants who took advantage of DCS, the types of specimens they submitted for testing, and testing results of these specimens from MX908 and FTS. Our findings indicated that most specimens provided by participants for testing were opioids and the presence of fentanyl/fentanyl analogues was detected by the MX908 in more than half of the specimens believed to be heroin among participants. Agreement of testing results between MX908 and FTS in detection of fentanyl/fentanyl analogues was modest. Additional studies are needed to assess the implementation and use of MX908 in community-based and harm reduction-focused DCS.

\section{List Of Abbreviations}




\begin{tabular}{ll} 
Abbreviation & Definition \\
\hline FTS & Fentanyl testing strips \\
PWUD & People who use drugs \\
SSP & Syringe Services Program \\
\hline MX908 & MX908 Portable Mass Spectrometer \\
\hline DCS & Drug Checking Services
\end{tabular}

\section{Declarations}

\section{Ethics approval and consent to participate}

This study was approved by the Tufts University Health Sciences Institutional Review Boards.

\section{Consent for publication}

Not applicable.

\section{Availability of data and materials}

The datasets for the current study are not publicly available due to privacy concerns but may be available from the corresponding author on reasonable request and pending the approval of the RIZE Massachusetts Foundation and the Kraft Center for Community Health.

\section{Competing interests}

The authors declare that they have no competing interests.

\section{Funding}

Funding from the RIZE Massachusetts Foundation and the Kraft Center for Community Health supported this research (PI: Stopka). The funders had no role in the design of the study, data collection, analysis, and interpretation or preparation of the manuscript.

\section{Authors' contributions}

All authors wrote the main manuscript text, tables, and reviewed the manuscript.

\section{Acknowledgements}

The authors wish to thank the SSP staff who provided access to the DCS data, as well as information on the overall testing approach and experiences.

\section{References}

1. Ciccarone D. Fentanyl in the US heroin supply: a rapidly changing risk environment. Int J Drug Policy. 2017;46:107-11 DOl: 10.1016/j.drugpo.2017.06.010. 
2. Ciccarone D. The triple wave epidemic: Supply and demand drivers of the US opioid overdose crisis. Int J Drug Policy. 2019;71:183-8 DOI: 10.1016/j.drugpo.2019.01.010.

3. CDC. Opioid overdose, understanding the epidemic. 2021 [updated March 19, 2020. Available from: https://www.cdc.gov/drugoverdose/epidemic/index.html.

4. Somerville NJ, O'Donnell J, Gladden RM, Zibbell JE, Green TC, Younkin M, et al. Characteristics of fentanyl overdose - Massachusetts, 2014-2016. MMWR Morb Mortal Wkly Rep. 2017;66(14):382-6 DOI: 10.15585/mmwr.mm6614a2.

5. Scholl L, Seth P, Kariisa M, Wilson N, Baldwin G. Drug and opioid-involved overdose deaths - United States, 2013-2017. MMWR Morb Mortal Wkly Rep. 2018;67(5152):1419-27 DOI:

10.15585/mmwr.mm675152e1.

6. Ahmad FB, Rossen LM, Sutton P. Provisional drug overdose death counts NCHS. 2021.

7. CDC. Overdose deaths accelerating during COVID-19: expanded prevention efforts needed. 2020 [Available from: https://www.cdc.gov/media/releases/2020/p1218-overdose-deaths-covid-19.html.

8. Association AM. Issue brief: drug overdose epidemic worsened during COVID pandemic 2021 [updated June 1, 2021.

9. Slavova S, Rock P, Bush HM, Quesinberry D, Walsh SL. Signal of increased opioid overdose during COVID-19 from emergency medical services data. Drug Alcohol Depend. 2020;214:108176 DOI: 10.1016/j.drugalcdep.2020.108176.

10. CDC NCfHS, Office of Communication Drug overdose deaths in the U.S. top 100,000 annually for immediate release: November 17, 2021. 2021 [Available from: https://www.cdc.gov/nchs/pressroom/nchs_press_releases/2021/20211117.htm.

11. Hedegaard H, Miniño AM, Warner M. Drug Overdose Deaths in the United States, 1999-2017. NCHS Data Brief. 2018(329):1-8.

12. Health MDoP. Data brief: opioid-related overdose: deaths among Massachusetts residents 2021 [Available from: https://www.mass.gov/doc/opioid-related-overdose-deaths-among-ma-residents-may2021/download.

13. Singh VM, Browne T, Montgomery J. The emerging role of toxic adulterants in street drugs in the US illicit opioid crisis. Public Health Rep. 2020;135(1):6-10 DOI: 10.1177/0033354919887741.

14. Lyden J, Binswanger IA. The United States opioid epidemic. Semin Perinatol. 2019;43(3):123-31 DOI: 10.1053/j.semperi.2019.01.001. 
15. Glick JL, Christensen T, Park JN, McKenzie M, Green TC, Sherman SG. Stakeholder perspectives on implementing fentanyl drug checking: results from a multi-site study. Drug Alcohol Depend. 2019;194:527-32 DOI: 10.1016/j.drugalcdep.2018.10.017.

16. Sherman SG, Park, J. N., McKenzie, M., Morales, K., Christensen, T., \& Green, T. C. FORECAST study summary report John Hopkins Bloomberg School of Public Health.; 2018.

17. Laing MK, Tupper KW, Fairbairn N. Drug checking as a potential strategic overdose response in the fentanyl era. Int J Drug Policy. 2018;62:59-66 DOI: 10.1016/j.drugpo.2018.10.001.

18. Goldman JE, Waye KM, Periera KA, Krieger MS, Yedinak JL, Marshall BDL. Perspectives on rapid fentanyl test strips as a harm reduction practice among young adults who use drugs: a qualitative study. Harm Reduct J. 2019;16(1):3 DOI: 10.1186/s12954-018-0276-0.

19. Peiper NC, Clarke SD, Vincent LB, Ciccarone D, Kral AH, Zibbell JE. Fentanyl test strips as an opioid overdose prevention strategy: findings from a syringe services program in the Southeastern United States. Int J Drug Policy. 2019;63:122-8 DOI: 10.1016/j.drugpo.2018.08.007.

20. Karamouzian M, Dohoo C, Forsting S, McNeil R, Kerr T, Lysyshyn M. Evaluation of a fentanyl drug checking service for clients of a supervised injection facility, Vancouver, Canada. Harm Reduct J. 2018;15(1):46 DOI: 10.1186/s12954-018-0252-8.

21. Green TC, Park JN, Gilbert M, McKenzie M, Struth E, Lucas R, et al. An assessment of the limits of detection, sensitivity and specificity of three devices for public health-based drug checking of fentanyl in street-acquired samples. Int J Drug Policy. 2020;77:102661 DOI: 10.1016/j.drugpo.2020.102661.

22. Ciccarone D, Ondocsin J, Mars SG. Heroin uncertainties: exploring users' perceptions of fentanyladulterated and -substituted 'heroin'. Int J Drug Policy. 2017;46:146-55 DOI:

10.1016/j.drugpo.2017.06.004.

23. Marshall BDL, Krieger MS, Yedinak JL, Ogera P, Banerjee P, Alexander-Scott NE, et al. Epidemiology of fentanyl-involved drug overdose deaths: a geospatial retrospective study in Rhode Island, USA. Int J Drug Policy. 2017;46:130-5 DOI: 10.1016/j.drugpo.2017.05.029.

24. Ti L, Tobias S, Lysyshyn M, Laing R, Nosova E, Choi J, et al. Detecting fentanyl using point-of-care drug checking technologies: a validation study. Drug Alcohol Depend. 2020;212:108006 DOI: 10.1016/j.drugalcdep.2020.108006.

25. Vandergrift GW, Gill CG. Paper spray mass spectrometry: a new drug checking tool for harm reduction in the opioid overdose crisis. J Mass Spectrom. 2019;54(9):729-37 DOI: 10.1002/jms.4431.

26. Harper L, Powell J, Pijl EM. An overview of forensic drug testing methods and their suitability for harm reduction point-of-care services. Harm Reduct J. 2017;14(1):52 DOI: 10.1186/s12954-017-0179-5. 
27. MX908 ${ }^{\mathrm{TM}}$. 908 Devices Inc. Boston, Massachusetts. [

28. Rapid Response ${ }^{T M}$. BTNX Corporation, Canada. [

29. Tupper KW, McCrae K, Garber I, Lysyshyn M, Wood E. Initial results of a drug checking pilot program to detect fentanyl adulteration in a Canadian setting. Drug Alcohol Depend. 2018;190:242-5 DOI: 10.1016/j.drugalcdep.2018.06.020.

30. Krieger MS, Goedel WC, Buxton JA, Lysyshyn M, Bernstein E, Sherman SG, et al. Use of rapid fentanyl test strips among young adults who use drugs. Int J Drug Policy. 2018;61:52-8 DOI: 10.1016/j.drugpo.2018.09.009.

31. Sherman SG, Morales KB, Park JN, McKenzie M, Marshall BDL, Green TC. Acceptability of implementing community-based drug checking services for people who use drugs in three United States cities: Baltimore, Boston and Providence. Int J Drug Policy. 2019;68:46-53 DOI: 10.1016/j.drugpo.2019.03.003.

32. Rhodes B, Costenbader B, Wilson L, Hershow R, Carroll J, Zule W, et al. Urban, individuals of color are impacted by fentanyl-contaminated heroin. Int J Drug Policy. 2019;73:1-6 DOI: 10.1016/j.drugpo.2019.07.008.

33. Fogger SA. Methamphetamine use: a new wave in the opioid crisis? J Addict Nurs. 2019;30(3):219-23 DOI: 10.1097/jan.0000000000000298.

34. Hainer R. Polysubstance use and stimulants: a dangerous fourth wave in the opioid crisis. Boston Medical Center: HealthCity Newsletter 2019.

35. Jenkins R. The fourth wave of the US opioid epidemic and its implications for the rural US: a federal perspective Preventive Medicine. In press.

36. Beletsky L, Davis CS. Today's fentanyl crisis: Prohibition's Iron Law, revisited. Int J Drug Policy. 2017;46:156-9 DOI: 10.1016/j.drugpo.2017.05.050.

37. Foglia R, Kline A, Cooperman NA. New and emerging opioid overdose risk factors. Curr Addict Rep. 2021:1-11 DOI: 10.1007/s40429-021-00368-6.

38. Barratt MJ, Bruno R, Ezard N, Ritter A. Pill testing or drug checking in Australia: Acceptability of service design features. Drug Alcohol Rev. 2018;37(2):226-36 DOI: 10.1111/dar.12576.

39. Karamouzian M, Papamihali K, Graham B, Crabtree A, Mill C, Kuo M, et al. Known fentanyl use among clients of harm reduction sites in British Columbia, Canada. Int J Drug Policy. 2020;77:102665 DOI: 10.1016/j.drugpo.2020.102665. 
40. Stein MD, Kenney SR, Anderson BJ, Bailey GL. Perceptions about fentanyl-adulterated heroin and overdose risk reduction behaviors among persons seeking treatment for heroin use. J Subst Abuse Treat. 2019;104:144-7 DOI: 10.1016/j.jsat.2019.07.006.

41. Mars SG, Ondocsin J, Ciccarone D. Sold as heroin: Perceptions and use of an evolving drug in Baltimore, MD. J Psychoactive Drugs. 2018;50(2):167-76 DOI: 10.1080/02791072.2017.1394508.

42. Daniulaityte R, Carlson RR, Juhascik MP, Strayer KE, Sizemore IE. Street fentanyl use: Experiences, preferences, and concordance between self-reports and urine toxicology. Int J Drug Policy. 2019;71:3-9 DOI: 10.1016/j.drugpo.2019.05.020. 